\title{
Erratum to: Characterization of the radiolabeled metabolite of tau PET tracer 18F-THK5351
}

\author{
Ryuichi Harada $^{1} \cdot$ Shozo Furumoto $^{2} \cdot$ Tetsuro Tago $^{2} \cdot$ Katsutoshi Furukawa $^{3}$. \\ Aiko Ishiki $^{3}$ - Naoki Tomita ${ }^{3}$ - Ren Iwata ${ }^{2}$ Manabu Tashiro ${ }^{2}$ - Hiroyuki Arai ${ }^{3}$. \\ Kazuhiko Yanai $^{2,4} \cdot$ Yukitsuka Kudo $^{1,2}$ - Nobuyuki Okamura ${ }^{1,2,5}$
}

Published online: 13 October 2016

(C) Springer-Verlag Berlin Heidelberg 2016

Erratum to: European Journal of Nuclear Medicine and Molecular Imaging 43(12):2211-2218

DOI 10.1007/s00259-016-3453-y

The name of Katsutoshi Furukawa was swapped in the original version of this paper. Correct name is presented here.

The online version of the original article can be found at http://dx.doi. org/10.1007/s00259-016-3453-y.

\footnotetext{
Nobuyuki Okamura

nookamura@med.tohoku.ac.jp

1 Division of Neuro-imaging, Institute of Development, Aging and

Cancer, Tohoku University, Sendai 980-8575, Japan

2 Cyclotron and Radioisotope Center, Tohoku University, Sendai 980-8578, Japan

3 Department of Geriatrics and Gerontology, Institute of Development, Aging and Cancer, Tohoku University, Sendai 980-8575, Japan

4 Department of Pharmacology, Tohoku University School of Medicine, Sendai 980-8575, Japan

5 Division of Pharmacology, Faculty of Medicine, Tohoku Medical and Pharmaceutical University, Sendai 981-8558, Japan
} 